\title{
Development of the Kyoto University Rare Materials Digital Archive
}

\author{
Chifumi Nishioka *
}

\begin{abstract}
In this article, we describe the Kyoto University Rare Materials Digital Archive, which has been officially launched on December 1, 2017. As of January 29, 2019, the digital archive provides 12,793 titles, including historically important manuscripts, maps, and drawings. The digital archive has been developed to enhance resources for research in humanities, supported by the Open Access promotion project of Kyoto University. The digital archive is compatible with the IIIF (International Image Interoperability Framework), which provides a set of protocols to promote interoperable use of images over different institutions. Exploiting the IIIF, we have launched collaborations with external institutions. In order to understand to which extent the digital archive has been used, we compute the usage statistics using server logs recorded to the digital archive. The usage statistics reveals that the number of accesses to the digital archive has increased since its launch. Almost $60 \%$ of accesses come from Japan. Regarding accesses from foreign countries, we see a lot of accesses from China, the United States, and other Asian countries. In terms of influence from collaborations with external institutions, we do not see a significant increase of accesses to materials in the field of these collaborations. In order to promote the use of outcomes of the collaborations, we should reach to researchers and disseminate the outcomes to them.
\end{abstract}

Keywords: digital archive, digital library, open access, interoperability, collaboration

\section{Introduction}

Open access to scholarly articles refers to the removal of barriers including price barriers and permission barriers (i e.e, copyright and licensing restrictions) from accessing them [1]. Over the decades, the Open Access movement has evolved with the support by academic communities and different stakeholders [2]. Following this movement, Kyoto University adopted the Kyoto University Open Access policy ${ }^{1}$ in 2015. The policy mandates faculty members of Kyoto University to make their journal

\footnotetext{
* Kyoto University Library, Kyoto, Japan

${ }^{1}$ http://www.kulib.kyoto-u.ac.jp/uploads/oapolicy.pdf, last accessed on 02/27/2019
} 
articles public on the web using our institutional repository KURENAI (Kyoto University Research Information Repository $)^{2}$. Furthermore, we have launched the Open Access promotion project ${ }^{3}$ in 2016. The Open Access promotion project is a part of Kyoto University Priority Strategic Action Plan (2016-2021) focusing on the third midterm objectives, in order to realize "WINDOW: A Vision for the Future" aims to enhance our capabilities of supporting research by taking the following actions:

(1) promote open access to research outcomes (e .g., journal articles) through our institutional repository KURENAI

(2) digitize our rare materials, which include national treasure, important cultural properties, and other historically important manuscripts, maps, and drawings, and make them open access in the Kyoto University Rare Materials Digital Archive, in order to improve availabilities of resources for research in humanities and social sciences

(3) distribute the above-mentioned scholarly contents internationally

(4) develop human resources for the management of research data including open access in accordance with open science and research integrity

(5) organize a project team led by experts and train research support staffs systematically

In this article, we describe the development of the Kyoto University Rare Materials Digital Archive ${ }^{5}$, which advances open access of resources for research in humanities as pointed by (2). In the past 20 years, we have digitized rare materials and delivered them on different websites. To disseminate the digital images more efficiently, we officially launched the digital archive on December 1, 2017. It supports the IIIF (International Image Interoperable Framework) ${ }^{6}$, which enables interoperable use of images over different institutions by defining a couple of common APIs. Thus, the images available on the digital archive can be accessed and reused through APIs.

The reminder of this article is organized as follows: Section 2 provides the details of the digital archive including the description of IIIF, system configuration, and functions. Thereafter, Section 3 introduces two cases of collaborations with external institutions that exploit the IIIF. Section 4 shows the usage statistics of the digital archive, in order to understand how the digital archive has been used by users. Some of the usage statistics are also shown in the previous works $[3,4]^{7}$. Different from the previous works, we evaluate to which extent collaborations with other institutions influence usage of rare materials. Finally, Section 5 concludes this article.

\footnotetext{
${ }^{2}$ https://repository.kulib.kyoto-u.ac.jp/dspace/?locale=en, last accessed on 02/27/2019

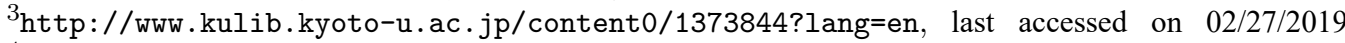

${ }^{4}$ https://www.kyoto-u.ac.jp/en/about/operation/window-a-vision-for-the-future.

$\mathrm{html}$, last accessed on 02/27/2019

${ }^{5}$ https://rmda.kulib.kyoto-u.ac.jp/

${ }^{6}$ http://iiif.io/

${ }^{7}$ The work [3] is a preprint version of the work [4].
} 


\section{Implementation of the Kyoto University Rare Materials Digital Archive}

This section describes the details of the Kyoto University Rare Materials Digital Archive, which has been launched on December 1, 2017. Regarding characteristics of the digital archive, it supports the IIIF, which enables interoperable use of images over institutions by defining common APIs. As of January 29, 2019, the digital archive stores 1,071,087 images of 12,793 titles.

Section 2.1 first introduces the IIIF. Thereafter, Section 2.2 briefly illustrates the system configuration of the digital archive. Section 2.3 describes interfaces as well as functions provided by the digital archive. Finally, Section 2.4 introduces the conditions of the use, by which users can freely reuse the digitized images in the digital archive.

\subsection{IIIF (International Image Interoperable Framework)}

Over the decades, a lot of institutions have digitized rare materials and made them public on the web. However, infrastructures to store images and web applications to deliver them have remained institution-specific. Thus, researchers who study images held by different institutions must switch from website to website, and must learn different interfaces and tools specific to each website. The IIIF (International Image Interoperable Framework) has been launched to address these challenges in 2012 [5]. So far, the IIIF has defined four APIs, which are the Image API, Presentation API, Search API, and Authentication API. The Image API defines a web service that returns an image in response to a standard HTTP or HTTPS request. The URI specifies the region, size, rotation, quality characteristics, and format of the requested image. For instance, when using an image as a thumbnail, a small value is set as the size of the image in the URI. The Presentation API specifies a web service that returns JSON-LD structured documents, which are called as IIIF manifests. IIIF manifests describe the structure and layout of a digitized object. In other words, the Presentation API focuses on the metadata for driving a viewing experience including image sequence, display labels, and licensing [5].

\subsection{System Configuration}

The digital archive uses the Image API and Presentation API. Figure 1 illustrates the system configuration of the digital archive. The digital archive comprises two main components: a content management system (CMS) and image server. We use Drupal $^{8}$ and IIP Image server ${ }^{9}$ as CMS and image server, respectively. The CMS manages and keeps contents other than images, including website designs, metadata and introductions of the rare materials, and image viewers. IIIF manifests are automatically generated by a Drupal module based on metadata stored in the CMS. The CMS also provides a search function, which is powered by Apache Solr ${ }^{10}$. The image server stores images of the rare materials. The images are delivered to a viewer on the CMS through the IIIF Image API.

\footnotetext{
${ }^{8}$ https://www.drupal.org/, last accessed on 02/26/2019

${ }^{9}$ https://iipimage. sourceforge.io/documentation/server/, last accessed on 02/26/2019

${ }^{10}$ http: //lucene.apache.org/solr/, last accessed on 02/26/2019
} 
1. visit website and get manifest.json

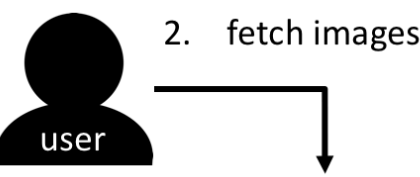

\begin{tabular}{|l|}
\multicolumn{1}{c|}{ CMS (Drupal) } \\
- Website design, Wireframe \\
- Vietadata \\
- manifest.json generator \\
\hline
\end{tabular}

\section{Image Server (IIP Image Server)}

- Image files (tif)

Figure 1: System configuration of the digital archive

\subsection{Interfaces and Functions}

This section introduces interfaces and functions of the digital archive.

Top page. Figure 2 is a screenshot of the top page of the digital archive. "Pick Up" in the middle of Figure 2 provides several categories, in which important or/and popular rare materials are classified. Collections are given in "Collection" of Figure 2 in the bottom. As of February 27, 2019, the digital archive provides eight pick ups and 34 collections. A user can switch languages with the toggle displayed in the upper right.

Bibliography page and image viewers Figure 3 shows a screenshot of a bibliography page. It provides the bibliographical information including record identifier, title, publication year, and notes. Universal Viewer ${ }^{11}$, which is one of the most popular IIIF-compatible image viewers, is embedded in the top of the page. Thus, a user can look through images on a bibliography page. In the bottom of the embedded image viewer, there are three icons, which are IIIF manifest, Universal Viewer, and Mirador. The icon of IIIF manifest provides a link to the IIIF manifest file of the corresponding bibliography. By clicking the icon of Universal Viewer or Mirador, a user can open the images on the corresponding image viewer. Mirador ${ }^{12}$ is another popular IIIFcompatible image viewer among the IIIF community. Compared to Universal Viewer, Mirador implements different functions to support researchers, such as comparison of images and annotations. We support both of the two image viewers, since they are the most popular ones among the IIIF community. Due to the simplicity of Universal Viewer, we choose Universal Viewer as the default viewer (i .e., image viewer embedded in the bibliography page).

Search The digital archive provides a search function ${ }^{13}$. Users can enter an arbitrary search query in the search box, in order to find materials. The target of the search

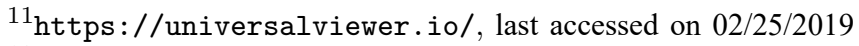

${ }^{12}$ http://projectmirador.org/, last accessed on 02/25/2019

${ }^{13}$ https ://rmda.kulib.kyoto-u.ac.jp/en/search, last accessed on 02/27/2019
} 


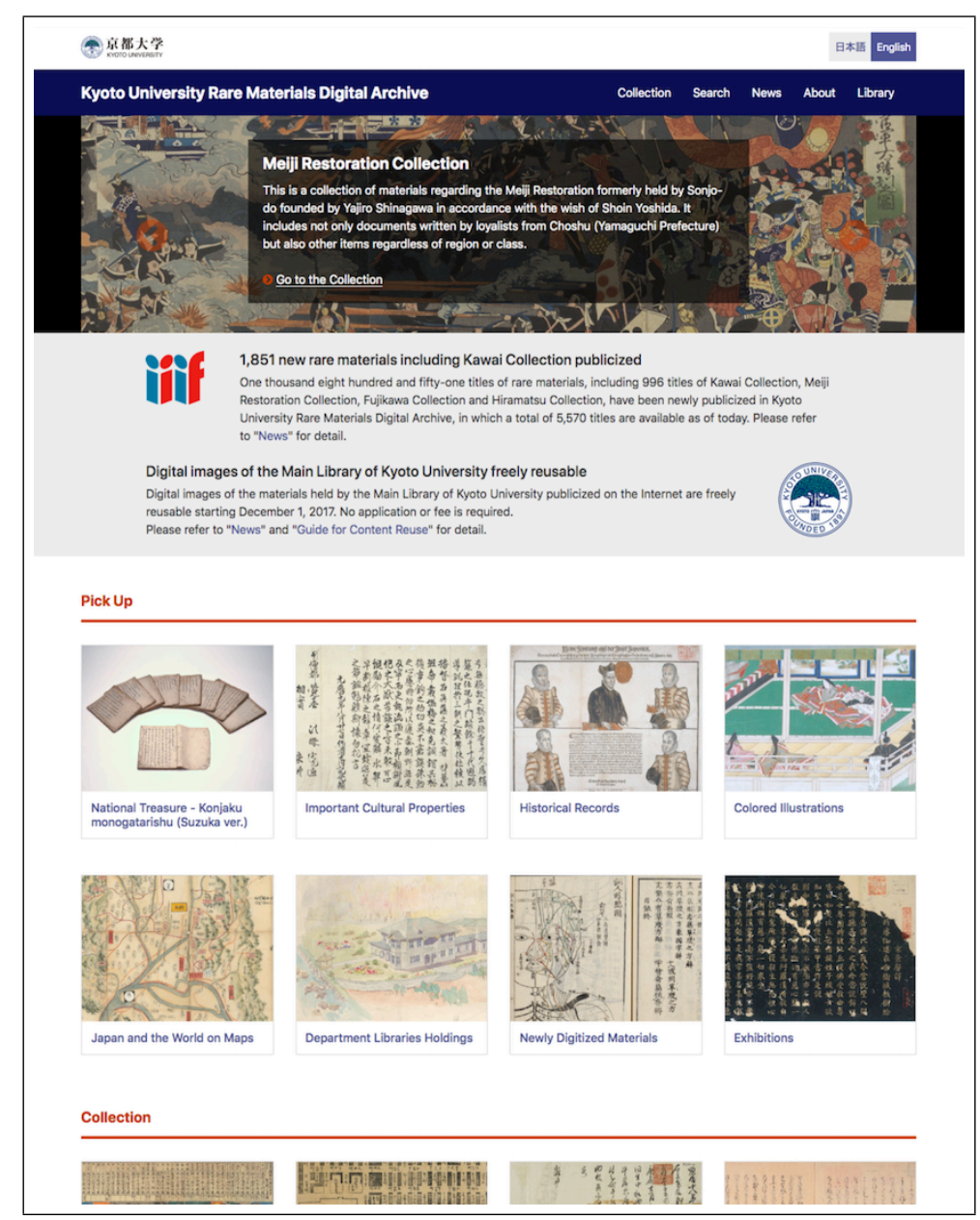

Figure 2: Top page of the digital archive

function is metadata of materials such as titles and authors and transcription text.

Links from OPAC A user can reach to the digitized rare materials not only from the digital archive but also from KULINE ${ }^{14}$, which is an OPAC (Online Public Access Catalog) of Kyoto University. As shown by the red rectangular in Figure 4, the bibliography page on KULINE provides a link to the corresponding bibliography page on the digital archive. KULINE and the digital archive store the identical metadata of the rare materials. KULINE enables users to find rare materials using advanced search, which is not given by the digital archive.

\subsection{Conditions of the Use}

To encourage academic communities to use images provided by the digital archive, we have revised the conditions of the use ${ }^{15}$ on December 1, 2017. Unless specifically

\footnotetext{
${ }^{14}$ https://kuline.kulib.kyoto-u.ac.jp/?lang=english

${ }^{15}$ https://rmda.kulib.kyoto-u.ac.jp/en/reuse, last accessed on 02/27/2019
} 


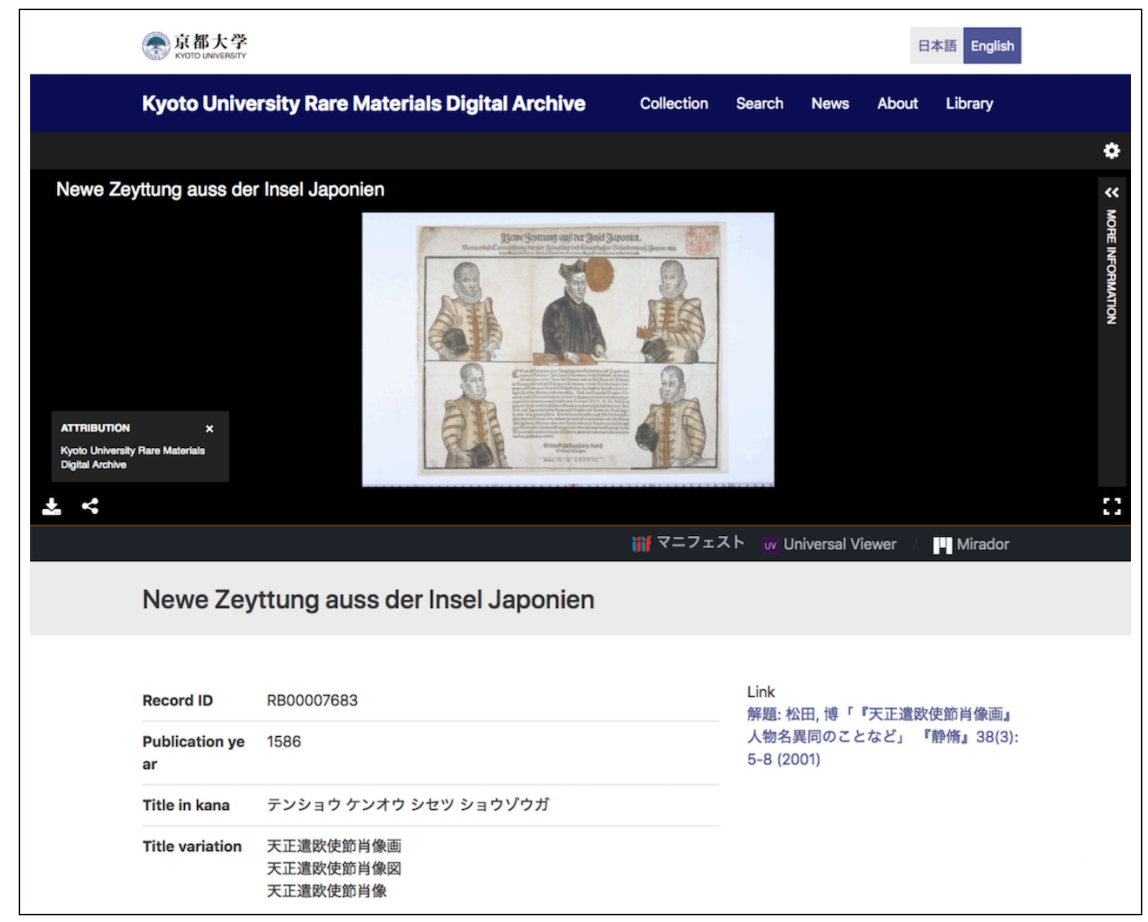

Figure 3: Bibliography page of the digital archive

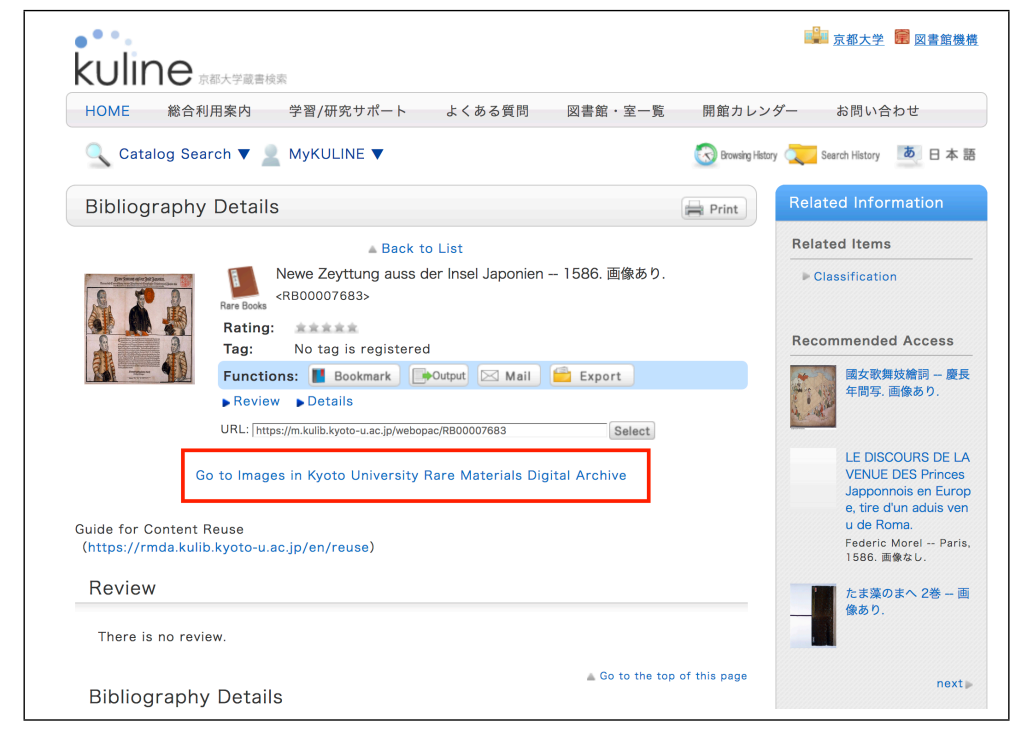

Figure 4: A Link to the digital archive from KULINE (OPAC) 
stated, the images owned by the Main Library, the Yoshida-South Library, and the Libraries of the Graduate School of Science of Kyoto University are available for any users to copy, adapt, or redistribute in any medium without application or fee under the following conditions:

1. indicate which libraries hold the original materials of the images

2. provide a link to the original image, if it is used on a website

3. indicate if changes are made to the images

We expect that the revision of the conditions of the use contributes to distribute our materials widely and promote researchers to study in them.

\section{Collaboration with External Institutions}

This section describes two cases in which rare materials from different institutions are integrated through the IIIF. First, Section 3.1 describes a collaborative project "Digital Fujikawa", where books of the Fujikawa collection are brought together exploiting the IIIF. Second, Section 3.2 presents mutual links between the SAT Daizōkyō database and the digital archive.

\subsection{Digital Fujikawa}

The Fujikawa collection is a collection of old medical books that had been collected by Dr. Yu Fujikawa (1865-1940). Books in the collection are stored in multiple institutions including Kyoto University and Keio University. In 2018, Kyoto University Library Network and Keio University Media Center have jointly launched a project "Digital Fujikawa -History of Medicine in Japan-". The project intends to contribute to the development of the research in the history of medicine in Japan by providing the comprehensive view of the Fujikawa collection that has been physically distributed over different institutions. On September 28, 2018, we have launched a website that virtually brings together the books of different institutions in one place using the IIIF. As of February 22, 2019, the website delivers 5,333 titles from three institutions including Kyoto University, Keio University, and the University of Tokyo.

Regarding the implementation of the website, we use Bootstrap ${ }^{16}$ and DataTables ${ }^{17}$. While a lot of libraries and museums implement websites for a collection based on a software such as Omeka ${ }^{18}[6,7]$, we do not use these kinds of softwares. Compared to implementations using softwares, our implementation has less functions but is light-weight and easy to maintain. We refer to the literature [8] for more details regarding our implementation.

Figure 5 shows the screenshot of the top page of the Digital Fujikawa. Bibliographies included in the Fujikawa collection is listed in a table. Each row in the table includes title, kana of the title (i .e., Japanese syllabary), romanization of the title, authors, published year, and icons of the IIIF manifest, Universal Viewer, Mirador,

\footnotetext{
${ }^{16}$ https://getbootstrap.com/, last accessed on 02/26/2019

${ }^{17}$ https://datatables.net/, last accessed on $02 / 26 / 2019$

${ }^{18}$ https: //omeka.org/, last accessed on 02/26/2019
} 


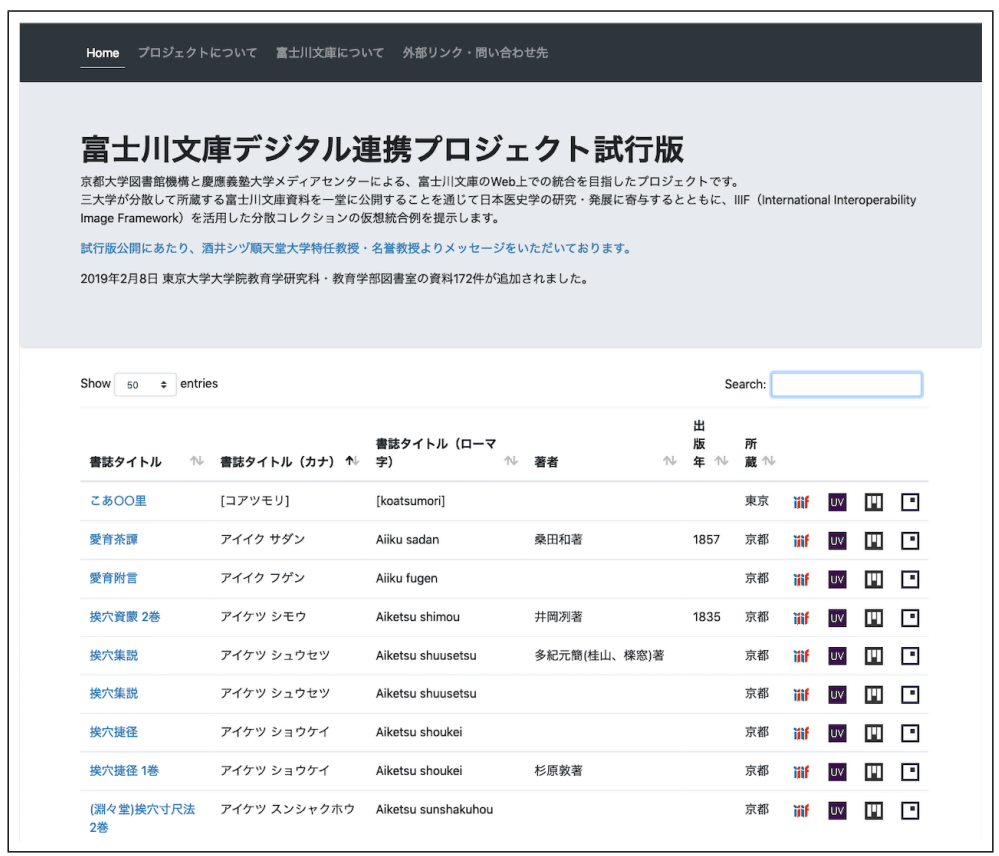

Figure 5: Top page of the Digital Fujikawa

and IIIF Curation Viewer. As all of the participating institutions intend to distribute their materials internationally, we have romanized titles for all books. Regarding image viewers, the Digital Fujikawa provides three options, which are Universal Viewer, Mirador, and IIIF Curation Viewer. IIIF Curation Viewer ${ }^{19}$ is an image viewer, where users can create curations across different IIIF-compatible digital archives [9]. The curations can be shared publicly. The Digital Fujikawa implements a search function, which is provided by DataTables. Users can search books using a search box located on the top right of the table. In addition, the Digital Fujikawa implements a sort function, which is provided by DataTables as well. Users can sort rows of the table by each column except icons.

In the future, we increase digital images available and participating institutions and develop new functions, in order to progress this project from the current trial phase to a full-scale phase by the end of academic year 2020/2021.

\subsection{Mutual Links to the SAT Daizōkyō Database}

On September 7, 2018, we have added mutual links between the SAT Daizōkyō database $[10,11]$ and the digital archive. The SAT Daizōkyō database is a full text search service for texts of 85 volumes of the Taishō Shinshū Daizōkyō provided by the SAT Daizōkyō Text Database Committee ${ }^{20}$. Each bibliography page of Buddhist study material in the digital archive has a link to a text of the corresponding material in the SAT Daizōkyō database, as shown by the red rectangular in Figure 6.

The SAT Daizōkyō database exploits metadata which have been added by researchers on the IIIF Manifests for Buddhist Studies21 [12]. The IIIF Manifests for Buddhist Studies

\footnotetext{
${ }^{19}$ http://codh.rois.ac.jp/software/iiif-curation-viewer/, last accessed on 02/26/2019

${ }^{20}$ http://21dzk.1.u-tokyo.ac.jp/SAT/members_en.html, last accessed on 02/27/2019
} 


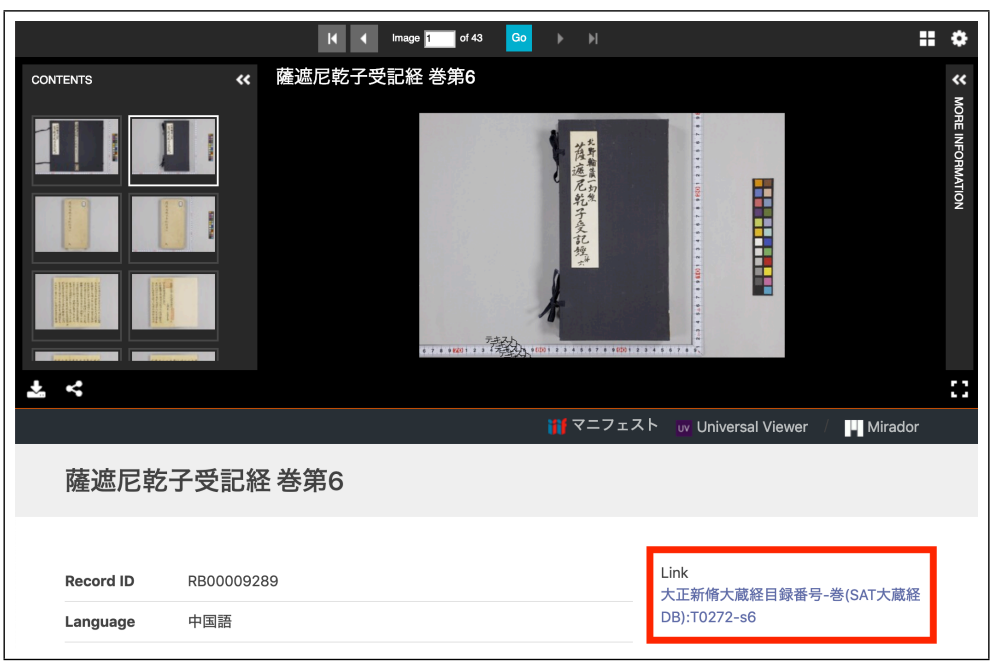

Figure 6: Bibliography page with a link to the SAT Daizōkyō Database

is a platform that collects 6,849 IIIF manifests ${ }^{22}$ regarding Buddhist study materials from a lot of different institutions including National Library of France, Harvard University, and Bavarian State Library. In the field of Buddhist studies, it has been practiced for a long time to assign catalog numbers to each material so that different versions of materials can be compared. Researchers can register IIIF manifests on the platform. In addition, they can browse images of Buddhist study materials on the platform and add catalog number, volume number, and line number to them. These added metadata is used to generate mutual links between the SAT Daizōkyō database and the digital archive. In addition, using these added metadata, researchers can easily find different versions of a Buddhist study material and text of the corresponding material. This case shows a pathway in which users provide added values to materials and the added values are returned to primarily publishing institutions [12].

\section{Usage Statistics of the Kyoto University Rare Materials Digital Archive}

This section reports the usage statistics of the digital archive, in order to understand to which extent the digital archive has been used. We calculate the usage statistics using the Japanese version of Advanced Web Statistics 7.7 (AWStats 7.7) ${ }^{23}$. Since AWStats has been used to compute the usage statistics of different digital libraries [13], we use it in this article. The AWStats analyzes access logs recorded on the server of the digital archive from October 4, 2017 to November 30, 2018.

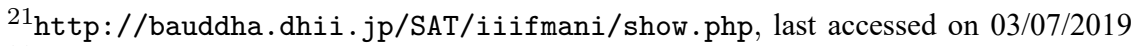

${ }^{22}$ The number is as of $03 / 07 / 2019$.

${ }^{23}$ http: //hobbit.ddo.jp/html/awstats.html\#DOWN, last accessed on 03/11/2019
} 


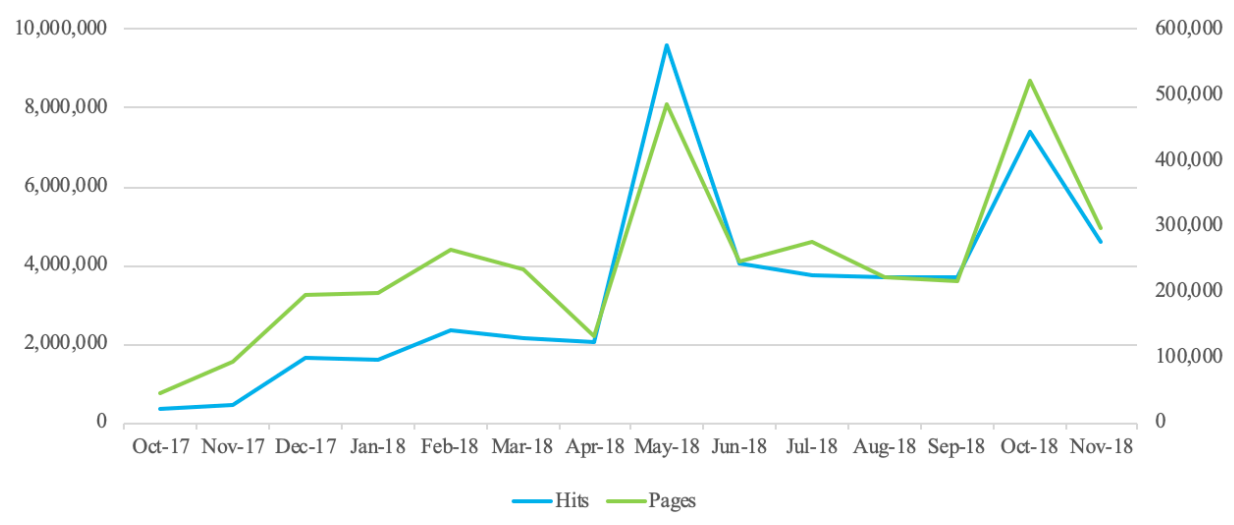

Figure 7: The numbers of pages and hits ${ }^{27}$

\subsection{Overview}

Figure 7 shows the numbers of pages and hits per month. The number of pages counts accesses to HTML, PHP, and ASP files. In contrast, the number of hits additionally counts accesses to other files such as CSS, image, and Java Script files, which are requested as a result of loading a page. Overall, we observe the increasing trend in both the numbers of pages and hits. There are spikes at May and October, 2018. Regarding the spike of May, 2018, it is caused by the newspaper article ${ }^{24}$ regarding the Fujikawa collection published by Kyoto Shimbun ${ }^{25}$. Since the newspaper article refers to Kaitai Shinsho ${ }^{26}$, it received a lot of accesses especially. In terms of the spike of October, 2018, it is made by a program which automatically collects contents of the digital archive.

Figure 8 presents the numbers of unique visitors and visits per month. We see the spike at May, 2018, which is caused by the newspaper article mentioned in the previous paragraph. We observe that both the numbers of visitors and visits after May, 2018 are larger than those before May, 2018. We assume that this is because that a part of users who first visited the digital archive due to the newspaper article have continued to access. Hence, being reported by a newspaper article might help to acquire new users.

Subsequently, we try to to investigate variables that influence the number of hits. A multiple linear regression is calculated to predict the number of hits for each date based on three variables: the number of materials published on the digital archive, the publication of news on the digital archive, and the mention by external media. Since we count the number of hits for each day for the analysis, we have 423 observations. For the publication of news on the digital archive, we set 1 if a news is released from the digital archive on a date. Otherwise, we set 0 . For the mention by external media, we set 1 if a news is released from external media on a date.

\footnotetext{
${ }^{24}$ https://www.kyoto-np.co.jp/top/article/20180513000109, last accessed on 01/02/2019

${ }^{25}$ A daily newspaper published in Kyoto, Japan

${ }^{26}$ It roughly means "New Text on Anatomy". It is a medical textbook translated into Japanese in the 18 th century.

${ }^{27}$ Shown in the previous works $[3,4]$ as well.

${ }^{28}$ Shown in the previous works $[3,4]$ as well.
} 


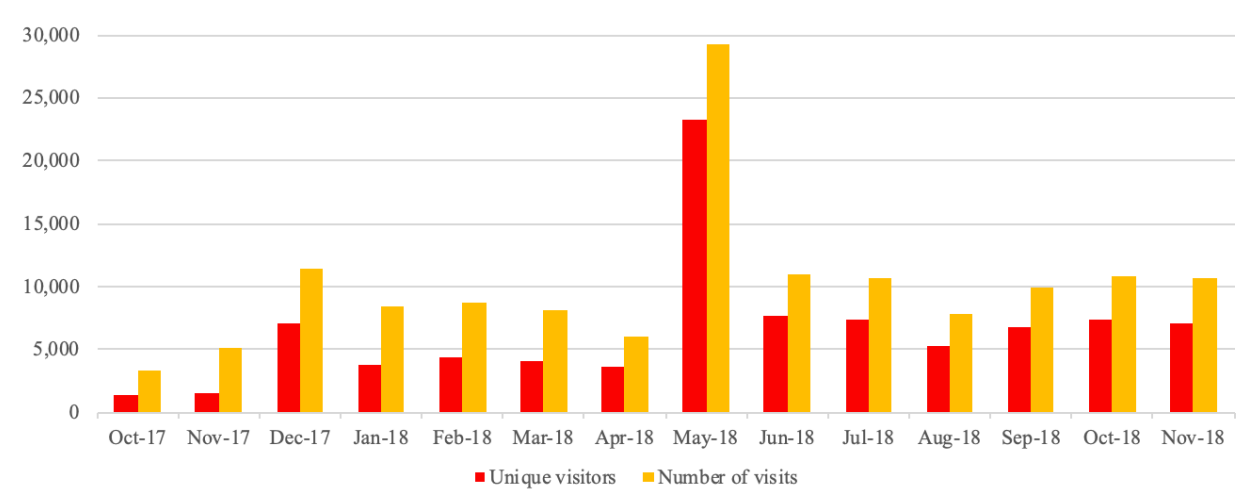

Figure 8: The numbers of unique visitors and visits ${ }^{28}$

Table 1: Coefficients calculated for the multiple regression analysis

\begin{tabular}{l|r|r}
\hline \multicolumn{1}{c|}{ Explanatory Variable } & Coefficient & t-value \\
\hline \hline The number of materials & 0.0478 & 7.007 \\
The publication of news on the digital archive & -0.0090 & -0.282 \\
The mention by external media & 0.0019 & 0.035 \\
\hline
\end{tabular}

We search for news using a couple of Japanese newspaper databases such as Nikkei Telecom ${ }^{29}$, Yomidasu Database Service ${ }^{30}$, and Maisaku ${ }^{31}$. However, we find only one article about Fujikawa collection released in May, 2 018. We normalize each variable before calculating the regression. A significant regression equation was found $\left(F(3,420)=16.40, p<0.001\right.$, with an $R^{2}$ of .105 . Table 1 provides coefficients of explanatory variables. We see that the number of materials have the largest influence on the number of hits. Regarding the other explanatory variables, we see relatively small influences. S ince $b$ oth the publication of $n$ ews a nd m ention by external media are not frequent cases, we need to observe more cases to draw a conclusion.

\subsection{Users}

This section reports the usage statistics focusing on users' attributions.

Table 2 presents the number of pages and hits per country. We identify countries from which the digital archive is accessed using IP addresses recorded in the server and the GeoIPfree plugin. As shown in Table 2, more than half of accesses come from Japan. Regarding accesses from foreign countries, we observe accesses from China, the United States, and other Asian countries including Singapore and Korea. The United States accounts for $14.18 \%$ of the number of pages, but it does for only about $3.30 \%$ regarding the number of hits. Since the number of hits tends to increase as users browse images on an image viewer, users from the United States browse images less compared to those from other countries. We observe that visitors from foreign countries access to rare materials related to foreign countries such as the

\footnotetext{
${ }^{29}$ https://telecom.nikkei.co.jp/, last accessed on 09/19/2019

30 https://database.yomiuri.co.jp/about/en/, last accessed on 09/19/2019

${ }^{31}$ https://mainichi.jp/contents/edu/maisaku/login.html, last accessed on 09/19/2019
} 
Table 2: The numbers of pages and hits per country ${ }^{34}$

\begin{tabular}{r|l|rr|rr}
\hline & \multicolumn{1}{|c|}{ Country } & \multicolumn{2}{|c|}{ Pages } & \multicolumn{2}{c}{ Hits } \\
\hline \hline 1 & Japan & $1,949,484$ & $56.94 \%$ & $32,803,316$ & $69.32 \%$ \\
2 & China & 558,471 & $16.31 \%$ & $7,610,003$ & $16.08 \%$ \\
3 & the United States & 485,459 & $14.18 \%$ & $1,560,651$ & $3.30 \%$ \\
4 & Unknown & 215,827 & $6.30 \%$ & $2,972,474$ & $6.28 \%$ \\
5 & Singapore & 57,058 & $1.67 \%$ & 83,764 & $0.18 \%$ \\
6 & Korea & 36,183 & $1.06 \%$ & 612,171 & $1.29 \%$ \\
7 & Taiwan & 21,106 & $0.62 \%$ & 333,142 & $0.70 \%$ \\
8 & Netherlands & 16,937 & $0.49 \%$ & 243,169 & $0.51 \%$ \\
9 & Germany & 10,051 & $0.29 \%$ & 155,193 & $0.33 \%$ \\
10 & Hong Kong & 9,916 & $0.29 \%$ & 212,430 & $0.45 \%$ \\
\hline
\end{tabular}

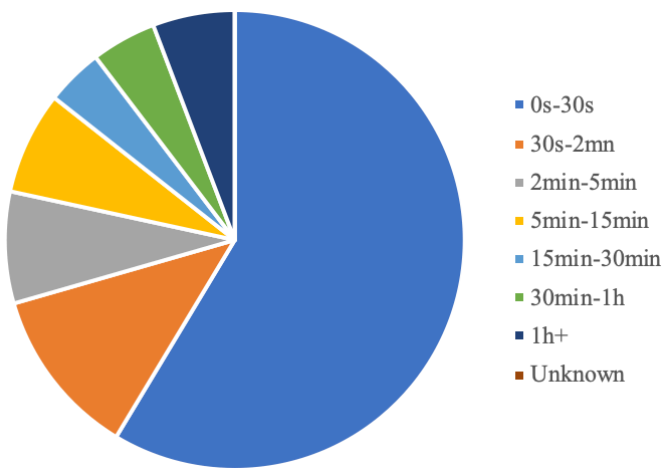

Figure 9: Visit durations ${ }^{35}$

Kawai Collection ${ }^{32}$ and the Architectural Drawings by Josiah Conder owned by the Faculty of Engineering, Kyoto University ${ }^{33}$.

Figure 9 illustrates distribution of visit durations. We see more than half of visitors stay at the digital archive for a short time (i e., less than 30 seconds). Visitors who stay for 30 minutes to an hour and longer than an hour account for $4.57 \%$ and $5.77 \%$, respectively. It indicates that more than 30 visitors per day stay at the digital archive for longer than 30 minutes, and more than 20 visitors browse the digital archive for more than an hour. We think they are not small numbers. The mean average of visit durations is 7 minutes 43 seconds.

\subsection{Functions}

This section presents the usage statistics of functions provided by the digital archive. Specifically, we report the usage statistics of the image viewers as well as search function.

\footnotetext{
${ }^{32}$ https://rmda.kulib.kyoto-u.ac.jp/en/collection/kawai, last accessed on 03/14/2019

${ }^{33} \mathrm{https}$ ://rmda.kulib.kyoto-u.ac.jp/en/collection/dep-arch-conder, last accessed on $03 / 14 / 2019$

${ }^{34}$ Shown in the previous works $[3,4]$ as well.

${ }^{35}$ Shown in the previous works $[3,4]$ as well.
} 
Table 3: The number of times each image viewer is used ${ }^{36}$

\begin{tabular}{l|c}
\hline \multicolumn{1}{c|}{ Image viewer } & Count \\
\hline \hline Universal Viewer & 84,022 \\
Mirador & 80,334 \\
\hline
\end{tabular}

Image viewers. As noted in Section 2.3, we implement two image viewers in the digital archive, which are Universal Viewer and Mirador. Table 3 shows the number of times a material is viewed on each image viewer. Please note that we count the number of clicks to icons of each image viewer (see Section 2.3 and Figure 3) as the number of times to be used. Thus, even if a user looks through different images on an image viewer, it is counted as one. As reported in Table 3, although Universal Viewers are used slightly more frequently than Mirador, both image viewers get almost same numbers of times to be used. Therefore, we think that it is reasonable to implement different image viewers on the digital archive and give an option to users to select an image viewer based on their purposes and preferences.

Search function. The digital archive provides search function ${ }^{37}$. Users can enter an arbitrary search query in the search box, in order to find materials whose bibliographic information and/or transcription text include the search query. 14,195 search queries have been used 56,557 times. Most search queries indicate titles of rare materials.

In addition, the English version of the digital archive gives search function ${ }^{38}$. At the English version, 695 search queries have been used 1,948 times. Since we get a lot of accesses from the Western countries such as the Uniter States as shown in Table 2, we expect that most search queries executed are in Latin characters. However, all of the 20 most frequent search queries are in Japanese or Chinese. Probably we should place the search box at a more descriptive place such as the top page as foreign libraries $\mathrm{do}^{39}$, in order to make the search box more accessible.

\subsection{Collaborations}

This section reports influence from each of the two collaborations described in Section 3 on usage statistics.

Digital Fujikawa Figure 10 shows the numbers of accesses to manifest and image files that belong to the Fujikawa collection per month. We observe spikes at May and October, 2018. Regarding the spike of May, 2018, it is due to the newspaper article mentioned in Section 4.1. We consider that a lot of users have accessed to the website of the Digital Fujikawa from its launch (i e., September 28, 2018) to the beginning of October, 2018, in order to just try the website. This caused the spike of October, 2018. Thereafter, the numbers of accesses to manifest as well as image files

\footnotetext{
${ }^{36}$ Shown in the previous works [3, 4] as well.

37 https ://rmda.kulib.kyoto-u.ac.jp/search, last accessed on 03/11/2019

${ }^{38}$ https://rmda.kulib.kyoto-u.ac.jp/en/search, last accessed on 03/11/2019

${ }^{39}$ For example, we find the search box at the top page in digital archives including Cambridge Digital Library (https://cudl.lib.cam.ac.uk/) and Bavarian State Library (https:// www.digitale-sammlungen.de/index $\cdot h t m l ? c=s t a r t s e i t e \& l=e n)$.
} 


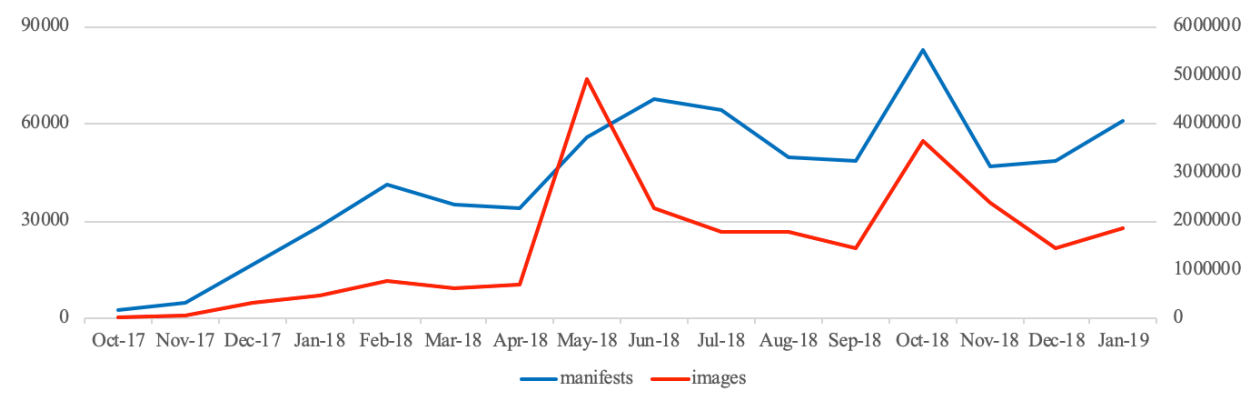

Figure 10: The numbers of accesses to manifest and image files that belong to the Fujikawa collection

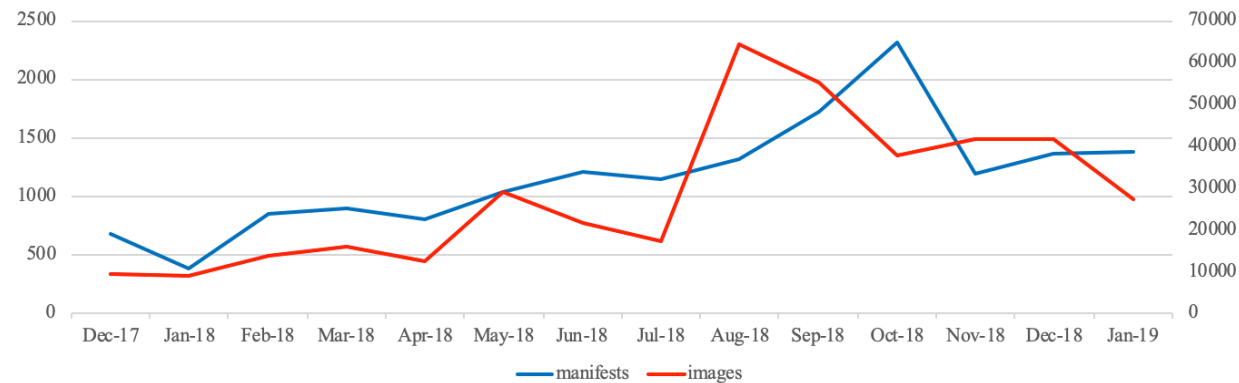

Figure 11: The numbers of accesses to manifest and image file with a link to the SAT Daizōkyō database

decline to December, 2018, but the numbers again increase at January, 2019. In order to promote the use of the digitized materials of the Fujikawa collection for research, we should reach to researchers in history of Japanese medicine and disseminate the website to them.

Mutual Links to the SAT Daizōkyō Database Figure 11 illustrates the numbers of accesses to manifest and image files whose corresponding bibliography is linked to the SAT Daizōkyō database. We observe that the numbers of accesses to manifest and image files after September, 2018 are consistently larger than those before September, 2018. Thus, we think that the mutual links increase accesses to the digitized materials regarding Buddhist studies.

\section{Conclusion}

This article introduces the development of the Kyoto University Rare Materials Digital Archive, which has been officially launched on December 1, 20 17. The digital archive has been implemented to make our holdings such as manuscripts open access and improve availabilities of resources for research in humanities. The digital archive is compliant with IIIF, which promotes interoperable use of images over different institutions. We describe collaborations with external institutions exploiting IIIF protocols, which are the Digital Fujikawa and mutual links to the SAT Daizōkyō database. 
Regarding the usage statistics of the digital archive, the number of accesses to the digital archive might have been influenced by events such as academic conferences or educational activities such as lectures. We will continue to observe the usage statistics and examine periodicities of the trend of the usage statistics in the future.

Since the purpose of the digital archive is to support researchers in humanities and to promote research in our digitized materials, we attempted to understand how many times our digitized materials are mentioned in scholarly articles published after 2017 using the CiNii Articles OpenSearch $\mathrm{API}^{40}$. However, the API did not cover scholarly articles published after 2017 yet, thus we could not investigate. We will continue to observe how the digital archive has been used and evaluate influence of the digitization of materials on research in humanities for a long time.

\section{Acknowledgments}

The author appreciates colleagues in the Library Planning Division and the Academic Support Division of Kyoto University Library for their works to develop and maintain the Kyoto University Rare Materials Digital Archive. The implementation of the Kyoto University Rare Materials Digital Archive has been supported by the Kyoto University Open Access promotion project.

The digitization of the Fujikawa collection in Kyoto University has been supported by the following projects and grants: (1 Grant for functional enhancements by the Ministry of Education, Culture, Sports, Science and Technology (MEXT,(2 Project to build an international collaborative research network for premodern Japanese texts (NIJL-NW project ${ }^{41}$ by the National Institute of Japanese Literature, and (3 Kyoto University Open Access promotion project.

\section{References}

[1] P. Suber, "Open access overview," 2004, [Online; accessed 10-March-2019]. [Online]. Available: https://legacy.earlham.edu/ peters/fos/overview.htm

[2] H. Piwowar, J. Priem, V. Larivière, J. P. Alperin, L. Matthias, B. Norlander, A. Farley, J. West, and S. Haustein, "The state of OA: a large-scale analysis of the prevalence and impact of open access articles," PeerJ, vol. 6, p. e4375, 2018. [Online]. Available: https://doi.org/10.7717/peerj.4375

[3] C. Nishioka, "Usage of Kyoto University Rare Materials Digital Archive - from October 4, 2017 to November 30, 2018-," Kyoto University Library, Tech. Rep., 2019, (In Japanese, The original title: 201710420181130 ) .[Online]. Available: http://hdl.handle.net/2433/237320

[4] - "Usage of Kyoto University Rare Materials Digital Archive in the first year," Journal of College and University Libraries, vol. 112, pp. 2038:1-2038:11, 2019, (In Japanese, The original title: IIIF ). [Online]. Available: https://doi.org/10.20722/jcul.2038

\footnotetext{
${ }^{40}$ https://ci.nii.ac.jp/info/en/api/a_opensearch_full.html, last accessed on 03/14/2019

${ }^{41}$ https://www.nijl.ac.jp/pages/cijproject/index_e.html, last accessed on 03/14/2019
} 
[5] S. Snydman, R. Sanderson, and T. Cramer, "The International Image Interoperability Framework (IIIF): A community \& technology approach for web-based images," in Archiving Conference. Society for Imaging Science and Technology, 2015, pp. 16-21. [Online]. Available: https://www.ingentaconnect. com/content/ist/ac/2015/00002015/00000001/art00005

[6] J. Kucsma, K. Reiss, and A. Sidman, "Using omeka to build digital collections: The METRO case study," D-Lib magazine, vol. 16, no. 3/4, pp. 1-11, 2010.[Online]. Available: https://doi.org/10.1045/march2010-kucsma

[7] J. L. Hardesty, "Exhibiting library collections online: Omeka in context," New Library World, vol. 115 , no. 3/4, pp. 75-86, 2014. [Online]. Available: http://dx.doi.org/10.1108/NLW-01-2014-0013

[8] C. Nishioka, "Reunion of the Fujikawa collection powered by IIIF," Computers and the Humanities Symposium "JinMonCom", vol. 2018, pp. 291-296, 2018, (In Japanese, The original title: IIIF.

[9] A. Kitamoto, J. Homma, and T. Saier, "IIIF Curation Platform: Next generation IIIF open platform supporting user-driven image sharing," in Computers and the Humanities Symposium "JinMonCom". Information Processing Society of Japan, 2018, pp. 327-334, (In Japanese, The original title: IIIF Curation Platform IIIF.

[10] K. Nagasaki, T. Tomabechi, and M. Shimoda, "Towards a digital research environment for Buddhist studies," Literary and linguistic computing, vol. 28, no. 2, pp. 296-300, 2013. [Online]. Available: https://doi.org/10.1093/1lc/fqs076

[11] S. D. T. D. Committee, "The SAT Daizōkyō Text Database," 2018, [Online; accessed 10-March-2019]. [Online]. Available: http://21dzk.lu-tokyo.ac.jp/SAT

[12] K. Nagasaki and M. Shimoda, "Possibilities of utilization of digital archives through open movement: A case study in the IIIF Manifests for Buddhist Studies," Computers and the Humanities Symposium "JinMonCom", pp. 389-394, 2018, (In Japanese, The original title: IIIF Manifests for Buddhist Studies.

[13] E. L. Black, "Web analytics: A picture of the academic library web site user," Journal of Web Librarianship, vol. 3, no. 1, pp. 3-14, 2009. [Online]. Available: https://doi.org/10.1080/19322900802660292 